\title{
High Mobility Organic Single-Crystal Transistors *
}

\author{
Masakazu Yamagishi, ${ }^{\dagger}$ Yukihiro Tominari, and Jun Takeya \\ Department of Chemistry, Graduate School of Science, \\ Osaka University, 1-1 Machikaneyama, Toyonaka 560-0043, Japan
}

(Received 26 September 2007; Accepted 8 January 2008; Published 25 January 2008)

\begin{abstract}
We report a series of our experiments using organic single crystals to reach the maximum performance intrinsic to the materials. A consequence of the experiments is that a good prescription for realizing high-mobility devices is to induce carriers in inner crystals to avoid scattering at the surfaces. Intrinsic-semiconductor character of the high-purity organic crystals favors thermal diffusion of the carriers into the crystals in the presence of weak gate-electric fields. [DOI: 10.1380/ejssnt.2008.21]
\end{abstract}

Keywords: Organic single-crystal transistor; Self-assembled monolayers (SAMs); Hall effect; Organic transistor; Field effect

\section{INTRODUCTION}

Developing high-mobility organic field-effect transistors (OFETs) is one of the key technologies that can extend a market of organic electronics, providing easy-to-fabricate switching components in fundamental logic circuits, for example [1-9]. In this presentation, we report a series of our experiments using organic single crystals to reach the maximum performance intrinsic to the materials. Our experiments features development of the organic singlecrystal devices [8], achievement of the bench-mark mobility of $30-40 \mathrm{~cm}^{2} / \mathrm{Vs}[2]$, establishment of Hall-effect measurement to directly estimate density of the electric-field induced carriers $[1,7]$, and evaluation of newly synthesized air-stable compounds [4]. From the above experiments, it turned out that intrinsic-semiconductor character of high-purity organic crystal semiconductor favors thermal diffusion of the carriers into the crystals in the presence of weak gate-electric fields, presenting basic physics for future technologies of organic "crystal electronics".

\section{EXPERIMENTAL}

\section{A. Crystal growth}

The rubrene single crystals used for active layers were grown by physical vapor transport. Commercial sublimed grade product of Aldrich 551112 were vaporized and crystallized. The resultant crystals were brought to the sublimation boat and were recrystallized, so that purer rubrene crystals can be grown. The other crystals used for the present experiments, such as newly synthesized oligocalcogenophene and 9,10-diphenylanthracene (DPA) crystals, were also grown by the same technique.

\footnotetext{
* This paper was presented at Handai Nanoscience and Nanotechnology International Symposium (Handai Nano 2007), Osaka University, Japan, 26-28 September, 2007.

$\dagger$ Corresponding author: yamamasa@chem.sci.osaka-u.ac.jp
}

\section{B. Device fabrication}

Picking up a thin crystal with the thickness of less than $1 \mu \mathrm{m}$, we attached it electrostatically to various gate insulators on which gold electrodes for each experiment were patterned with photolithography technique. An advantage of the technique is full variation of gate insulating layers and of the organic semiconductor materials as long as they grow into thin crystals. To fabricate several devices for the measurements, we prepared combinations of 1) rubrene single crystal/SAMs-coated $\mathrm{SiO}_{2}, 2$ ) oligothiophene single crystal $/ \mathrm{SiO}_{2}$ (without surface coverage), and 3) $\mathrm{SiO}_{2} /$ rubrene single crystal/DPA single-crystal insulator. In the last device, both the $\mathrm{SiO}_{2}$ layer and the thin platelet of the DPA crystal (laminated on the rubrene crystal) work as gate dielectrics, forming a double-gate organic single crystal transistor.

\section{Measurement method}

The transistor characteristics are measured using Agilent Technology E5270 semiconductor parameter analyzer with multiple Source Measure Units. In addition to usual transfer measurements, we performed the four-terminal measurements to exclude the contact resistances. The values of mobility are estimated using standard equations from increasing rate of conductivity as a function of gate voltage $V_{\mathrm{G}}$ [1-9]. To detect Hall effect, the transverse voltage $V^{\text {trans }}$ is monitored during $V_{\mathrm{G}}$ sweep and Hall coefficient $R_{\mathrm{H}}$ is given as $R_{\mathrm{H}}=\Delta V^{\text {trans }} / \Delta B I_{\mathrm{D}}(=1 / n e)$ where $B$ is magnetic field.

\section{RESULTS AND DISCUSSIONS}

\section{A. SAM-coated $\mathrm{SiO}_{2} /$ rubrene crystal transistors}

We first preparesd alkyl fluoride terminated selfassembled monolayers (F-SAMs) on the $\mathrm{SiO}_{2}$ surfaces. Figure 1 shows transfer characteristics of the singlecrystal transistor with the F-SAMs that exhibited the highest mobility. Taking the slope of the curvature in Fig. 1(a), results of two-terminal measurement, $\mu^{2 \mathrm{~T}}$ is estimated as $\sim 18 \mathrm{~cm}^{2} / \mathrm{Vs}$ when $-2 \mathrm{~V}<V_{\mathrm{G}}<0.5 \mathrm{~V}$, whereas $\mu^{2 \mathrm{~T}} \sim 5 \mathrm{~cm}^{2} /$ Vs when $V_{\mathrm{G}}<-4$ V. In Fig. 1(b), results of the four-terminal measurement are shown for 

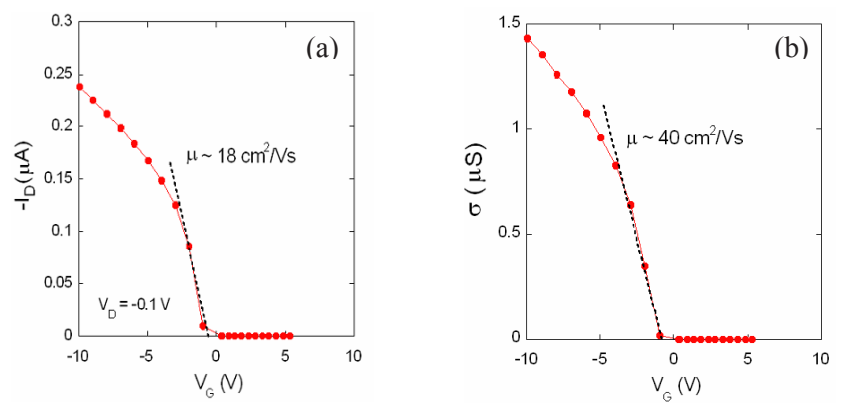

FIG. 1: Transfer characteristics of the single-crystal rubrene field-effect transistor that exhibits the highest mobility. (a) $I_{\mathrm{D}}$ and (b) $\sigma$ as function of $V_{\mathrm{G}}$.

the same sample. The similar "convex" curvature appears for the two-dimensional conductivity $\sigma$. Therefore, the slope change in the transfer characteristics is attributed not to the electric contacts to the electrodes, as was reported for thin-film devices in the past, but to an intrinsic property of the channel conductivity in the rubrene semiconductor channels. The four-terminal mobility $\mu^{4 \mathrm{~T}}$ reaches $\sim 40 \mathrm{~cm}^{2} / \mathrm{Vs}$ when $-2 \mathrm{~V}<V_{\mathrm{G}}<-0.5 \mathrm{~V}$, though it reduces to $\sim 15 \mathrm{~cm}^{2} / \mathrm{Vs}$ when $V_{\mathrm{G}}<-4 \mathrm{~V}$. For the statistics of similarly prepared 5 samples, $\mu^{4 \mathrm{~T}}$ is in the range of $25-40 \mathrm{~cm}^{2} / \mathrm{Vs}$ at the low $V_{\mathrm{G}}$ and of $8-10 \mathrm{~cm}^{2} / \mathrm{Vs}$ at the high $V_{\mathrm{G}}$. Mobility values estimated from the saturation current in the output characteristics are $\sim 15 \mathrm{~cm}^{2} / \mathrm{Vs}$ which is identical to $\mu^{4 \mathrm{~T}}$ when more than $-4 \mathrm{~V}$ is applied to the gate electrode. We note that relatively large $V_{\mathrm{D}}$ and $V_{\mathrm{G}}$ are necessary to saturate $I_{\mathrm{D}}$.

We also measured the Hall effect of similarly prepared devices. Figure 2 shows conductivity of the devices with various SAMs as function of inverse Hall coefficient $R_{\mathrm{H}}^{-1}(=n e)$; Samples $\mathrm{A}$ and $\mathrm{B}$ incorporate F-SAMs and alkyl terminated SAMs $\left(\mathrm{CH}_{3}-\mathrm{SAMs}\right)$ prepared in a dry glove box, respectively. Samples $\mathrm{C}$ and D incorporate FSAMs and $\mathrm{CH}_{3}-\mathrm{SAM}$ s prepared in ambient atmosphere. As compared with the device without the SAM treatment (E), the devices with SAMs $(\mathrm{A} \sim \mathrm{D})$ show higher conductivity in general. From the slope of the curves, we estimated $\mu$ as $\mu=d \sigma / d(n e)$, so that the dry-SAM device (A and B) shows the highest mobility of $\sim 35 \mathrm{~cm}^{2} / \mathrm{Vs}$ with the application of small gate voltages. These devices exhibit steeper slopes with smaller carrier density, though the slopes become less steep with largeer carrier density.

The simple electrostatic consideration gives a length scale $L_{a}$ which represents the effective thickness of the accumulation layer as function of $V_{\mathrm{G}}$, as a result of competition between thermal diffusion of the field-injected carriers into the crystal and the gate electric field that attract them to the surface of the crystal. In the above simple model neglecting interface traps, $L_{a}$ is described as $L_{a}=\left(2 \varepsilon_{s} k T\right) / e C_{i}\left(V_{\mathrm{G}}-V_{\mathrm{th}}\right)$, where $\varepsilon_{s}$ is permittivity of the organic semiconductor and $k$ is Boltzmann constant [1]. Assuming that $\varepsilon_{s}$ is approximately 3 times as large as the permittivity of vacuum, $L_{a}$ is estimated to be $\sim 19 \mathrm{~nm}$ when higher mobility was obtained at low $V_{\mathrm{G}}$ $\left(-\left(V_{\mathrm{G}}-V_{\mathrm{th}}\right)<1 \mathrm{~V}\right)$. On the other hand, the mobility is reduced with diminishing $L_{a}$ at higher gate voltages when the carriers are confined to a few monomolecular layers at the surface of the crystal. The result suggests that car-

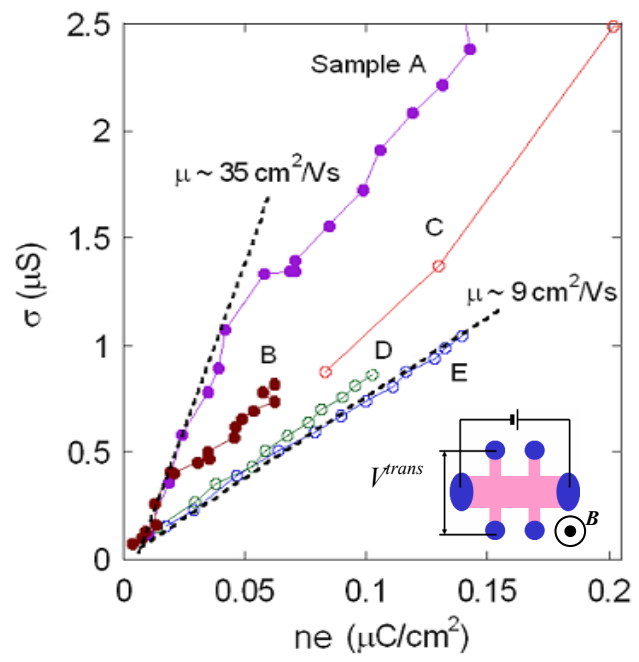

FIG. 2: $\sigma$ vs. ne plot. The dash line slopes estimate the mobilities.
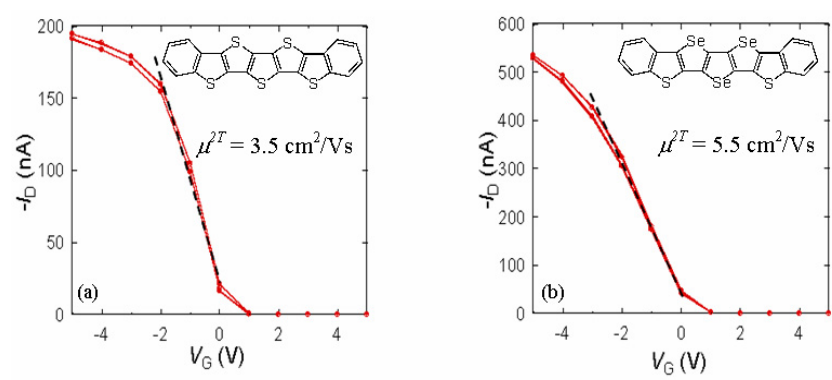

FIG. 3: Transfer characteristics of (a) oligothiophene and (b) oligoselenophene.

riers in the vicinity of the interface are more affected by the random potential of the amorphous gate insulator due to the increased scattering rate. In other words, the best mobility ever reported is attributed to the presence of the in-crystal carriers that are in the periodic arrangement of the ordered molecules.

\section{B. Newly synthesized air-stable oligocalcogenophene}

We fabricated new oligocalcogenophene crystal transistors and evaluated the two-terminal mobilities $\mu^{2 \mathrm{~T}}$ (Figure 3). Oligothiophene crystals show $\sim 3.5 \mathrm{~cm}^{2} / \mathrm{Vs}$ and oligoselenophene crystals show $\sim 5.5 \mathrm{~cm}^{2} / \mathrm{Vs}$ at low $V_{\mathrm{G}}$. These mobilities are rather high among organic semiconductors researched so far. Note that both calcogenophenes are extremely stable in air, keeping the same device performances for months.

\section{Double gate transistor with an insulating organic crystal}

From the above prescription that in-crystal carriers have higher mobility than those located at the crystalline surfaces, we attempted to distribute carriers deeper into the crystals, forming a double-gate FET structure; after constructing usual single-gate FETs with the 

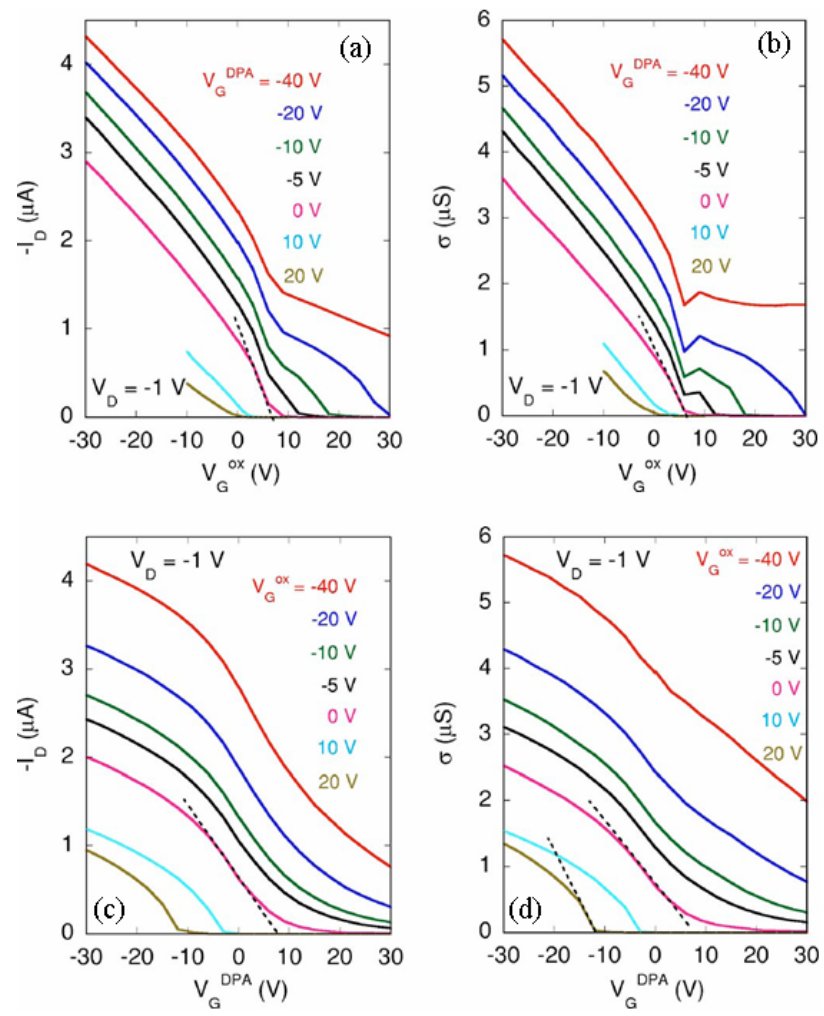

FIG. 4: Transfer characteristics of the double-gate rubrene single crystal transistor. (a) $I_{\mathrm{D}}$ and (b) $\sigma$ as function of sweeping $V_{\mathrm{G}}^{\mathrm{ox}}$. (c) $I_{\mathrm{D}}$ and (d) $\sigma$ as function of sweeping $V_{\mathrm{G}}^{\mathrm{DPA}}$. The dashed lines are guides to estimate mobility values.

rubrene/ $\mathrm{SiO}_{2}$ structure, a DPA gate-insulating crystal is attached to form the second transistor on the other surface of the thin rubrene crystal. Balancing the two gate voltages to extend the depletion layer in the middle of the crystal, the in-crystal carriers can be responsible for the conductivity. Figure 4 shows the transfer characteristics as functions of the gate voltage $V_{\mathrm{G}}^{\mathrm{ox}}$ in the $\mathrm{SiO}_{2}$ and that in the DPA $V_{\mathrm{G}}^{\mathrm{DPA}}$. From the slopes of Fig. $4(\mathrm{a})$ and (b), $\mu^{4 \mathrm{~T}}$ and $\mu^{2 \mathrm{~T}}$ are estimated to be $13-21 \mathrm{~cm}^{2} / \mathrm{Vs}$ and $3-7$ $\mathrm{cm}^{2} / \mathrm{Vs}$ depending on $V_{\mathrm{G}}^{\mathrm{ox}}$, respectively.

It is quite natural to assume that two conducting channels are formed near the top and bottom surfaces of the thin rubrene crystals when relatively high negative gate voltages are applied to the both surfaces because the gate electric fields attract holes to the surfaces. The device responds to $V_{\mathrm{G}}^{\mathrm{DPA}}$ and $V_{\mathrm{G}}^{\mathrm{ox}}$ independently as $\sigma=$ $\sigma^{\mathrm{DPA}}\left(V_{\mathrm{G}}^{\mathrm{DPA}}\right)+\sigma^{\mathrm{ox}}\left(V_{\mathrm{G}}^{\mathrm{ox}}\right)$, so that the transfer curves shown in each panels of Fig. 4 are parallel with each other in this case. On the other hand, when either $V_{\mathrm{G}}^{\mathrm{DPA}}$ and $V_{\mathrm{G}}^{\mathrm{ox}}$ is applied to deplete the hole carriers, the conductivity is not merely an additive of that of the DPA side $\sigma^{\mathrm{DPA}}\left(V_{\mathrm{G}}^{\mathrm{DPA}}\right)$ and that of the oxide side $\sigma^{\text {ox }}\left(V_{\mathrm{G}}^{\mathrm{ox}}\right)$ anymore; as observed in Fig. $4(\mathrm{~b}), \sigma\left(V_{\mathrm{G}}^{\mathrm{DPA}}=-40 \mathrm{~V}\right)-\sigma\left(V_{\mathrm{G}}^{\mathrm{DPA}}=-20 \mathrm{~V}\right)$ is still dependent on $V_{\mathrm{G}}^{\mathrm{ox}}$, for example. The observation gives evidence that the holes are exchanged from one side to the other through the inner crystal region. We therefore need to assume that the thickness $L_{\text {dep }}$ of the depletion layer is comparable to that of the crystal $(\sim 1 \mu \mathrm{m})$, so that the bottom-gate electric field extends into the crystal to partially remove holes that are supplied from the top-gate transistor. Simply estimating the ionic charge in the depletion layer as $L_{\text {dep }} N_{\text {imp }}$ per unit area $\left(N_{\text {imp }}\right.$ is the density of hole-trapping impurities), it can be noticed that only $3 \mathrm{~V}$ is enough for $V_{\mathrm{G}}^{\mathrm{ox}}$ to form the $1-\mu \mathrm{m}$ thick depletion layer, assuming that $N_{\mathrm{imp}} \sim 10^{15} \mathrm{~cm}^{3}$. Such a small number of the in-crystal trap density is consistent with the result of independent measurements of spacecharge-limited current in bulk rubrene crystals [10].

Interestingly, the transfer curves for $V_{\mathrm{G}}^{\mathrm{ox}}=20 \mathrm{~V}$ in Fig. 4(d) show the largest enhancement ratio of $\sigma$ as function of $V_{\mathrm{G}}^{\mathrm{DPA}}$ near the threshold of $V_{\mathrm{G}}^{\mathrm{DPA}}(\sim-12 \mathrm{~V}) \cdot \mu^{4 \mathrm{~T}}$ is evaluated to be high as $\sim 43 \mathrm{~cm}^{2} / \mathrm{Vs}$ under this condition, which is comparable to the benchmark results of the above SAMs OFETs. Since the in-crystal carriers are away from additional scattering by the gate insulators, such carriers can have higher mobility than those accumulated in the vicinity of the interfaces.

\section{CONCLUSION}

We have investigated variety of organic single-crystal transistors with different organic semiconductors, with different semiconductor-insulator interfaces, and of a double-gate FET structure. The bench-mark mobility of $30-40 \mathrm{~cm}^{2} / \mathrm{Vs}$ is realized, as the interface quality is maximized with SAMs deposited in a dry condition. The results of the Hall-effect measurements reasons that incrystal carriers avoiding a surface scattering are responsible when the gate electric field extends deeper into the crystal when it is not terminated by the interface traps. The observation suggests a prescription of developing high-mobility devices to make use of the in-crystal carriers of higher mobility. The $\mathrm{SiO}_{2} /$ rubrene crystal/DPA crystal double gate transistor is one possibility to distribute carriers in the crystal by controlling the thickness of the depletion layer. The double gate device indeed exhibits a very high-mobility of $\sim 43 \mathrm{~cm}^{2} / \mathrm{Vs}$ under the optimized condition.

\section{Acknowledgments}

This study is financially supported by Industrial Technology Research Grant Program in 2006 from New Energy and Industrial Technology Developing Organization (NEDO) of Japan. This study is also supported by a Grant-in-Aid for Scientific Research (Nos. 17069003, 18028029, and 19360009) from the Ministry of Education, Culture, Sports, Science, and Technology, Japan and Japan Science and Technology Agency of Osaka for the Seeds Inovating Project No. 11-054. 
196804 (2007).

[2] J. Takeya, M. Yamagishi, Y. Tominari, R. Hirahara, Y. Nakazawa, T. Nishikawa, T. Kawase, T. Shimoda, and S. Ogawa, Appl. Phys. Lett. 90, 102120 (2007).

[3] M. Yamagishi, J. Takeya, Y. Tominari, Y. Nakazawa, T. Kuroda, S. Ikehata, M. Uno, T. Nishikawa, and T. Kawase, Appl. Phys. Lett. 90, 182117 (2007).

[4] K. Yamada, T. Okamoto, K. Kudoh, S. Yamaguchi, and J. Takeya, Appl. Phys. Lett. 90, 072102 (2007).

[5] J. Takeya, K. Yamada, K. Hara, K. Shigeto, K. Tsukagoshi, S. Ikehata, and Y. Aoyagi, Appl. Phys. Lett. 88, 112102 (2006).
[6] K. Yamada, J. Takeya, K. Shigeto, K. Tsukagoshi, Y Aoyagi, and Y. Iwasa, Appl. Phys. Lett. 88, 122110 (2006).

[7] J. Takeya, K. Tsukagoshi, Y Aoyagi, T. Takenobu, and Y. Iwasa, Jpn. J. Appl. Phys. 44, L1393 (2005).

[8] J. Takeya, T. Nishikawa, T. Takenobu, S. Kobayashi, C. Goldmann, C. Krellner, T. Mitani. B. Batlogg, and Y. Iwasa, Appl. Phys. Lett. 85, 5078 (2004).

[9] J. Takeya, C. Goldmann, S. Haas, K. P. Pernstich, B. Ketterer, and B. Batlogg, J. Appl. Phys. 94, 5800 (2003).

[10] G. Horowitz, M. E. Hajlaoui, and R. Hajlaoui, J. Appl. Phys. 87, 4456 (2000). 